\title{
Die Rolle der Somatosensorik beim motorischen Lernen
}

\section{Carmen Krewer}

Spezifische sensorische Informationen können das motorische Lernen unterstützen. Das haben einige Studien gezeigt. Doch es funktioniert auch umgekehrt: Die primär motorische Intervention hat einen positiven Effekt auf die sensorische Wahrnehmungsleistung.

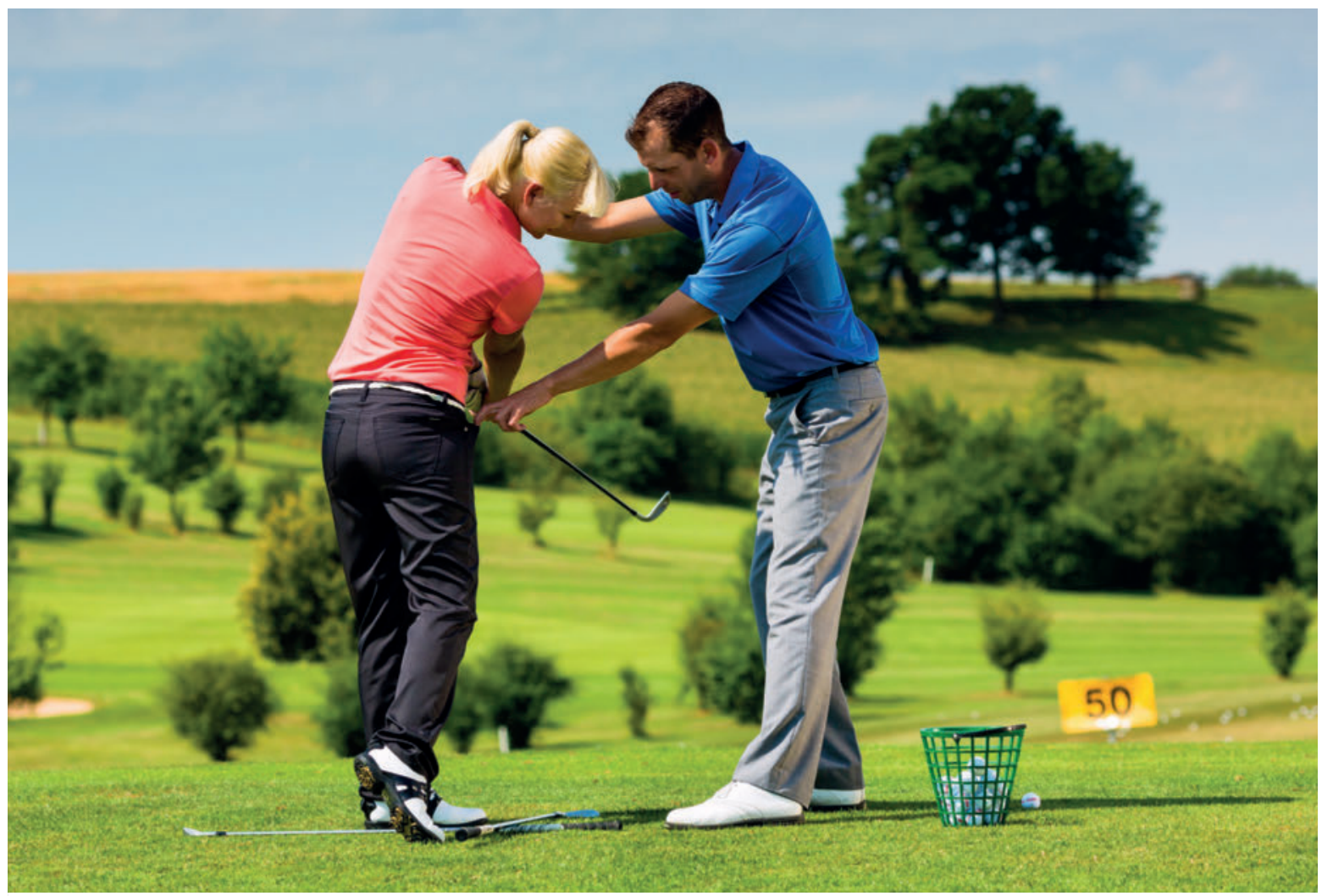

Abb. 1 Position und Bewegung sind im Sport und auch in der klinischen Forschung die beiden meistuntersuchten Sinnesmodalitäten der Propriozeption. (Quelle: Kzenon/stock.adobe.com; Symbolbild)

Unser Köper besitzt eine Vielzahl an sensorischen Systemen. Gardner und Johnson [7] unterscheiden sechs Sinnessysteme: das visuelle, das auditive, das vestibuläre, das somatosensorische, das gustatorische und das olfaktorische Sinnessystem. Zu allen diesen Sinnessystemen gehören spezifische Rezeptoren, die bei Stimulation Informationen an das zentrale Nervensystem senden, wo sie weiterverarbeitet werden. Die Wahrnehmung der Körperorientierung zur Erdvertikalen wird z. B. durch die Information von vestibulären, somatosensorischen und visuellen
Sensoren bestimmt [18]. Jeder dieser Sinne liefert dem zentralen Nervensystem unterschiedliche Informationen über die Orientierung im Raum oder auch der Körpersegmente zueinander.

Allerdings sind nicht alle sensorischen Informationen immer in gleicher Weise an einer Bewegung beteiligt, sondern können unter verschiedenen Bedingungen unterschiedlich gewichtet sein. Experimente von Lee und Aronson [14] z. B. konnten zeigen, dass Kleinkinder im 
Alter von 13-16 Monaten beim Stehen stärker auf visuelle Einflüsse reagieren als Erwachsene. Die Autoren führen dies u. a. auf eine noch limitierte Expertise das Stehen betreffend zurück. Kleinkinder zeigten als Reaktion auf den sogenannten „moving room“ (eine Bewegung der Seitenwände eines Raumes, die ein visuelles Fließmuster erzeugt, während die Versuchsperson auf einem stabilen, nicht bewegten Untergrund steht) eine stark ausgeprägte Körperschwankung, die in vielen Versuchen so ausgeprägt war, dass die Kinder sogar umfielen. Erwachsene schwankten in deutlich geringerem Ausmaß. Dies nimmt allerdings im höheren Lebensalter (d. h. 60 Jahre und älter) wieder zu. Durch alterskorrelierte Leistungseinbußen des somatosensorischen Systems, wie z. B. degenerative Veränderungen von peripheren Nervenfasern, orientieren sich die älteren Erwachsenen wieder stärker am visuellen Fließmuster [4].

Integrationsleistung Eine gute posturale Kontrolle verlangt also einen Abgleich der verschiedenen gleichgewichtsrelevanten Sinnessysteme miteinander und eine situationsabhängige Gewichtung. Diese Integrationsleistung ist abhängig von Übung und damit als Lernprozess zu verstehen [3]. Eine gute posturale Kontrolle ist die Basis für einen Großteil unserer motorischen Handlungen. Sowohl für die gleichgewichtsregulatorische Komponente jeder motorischen Handlung als auch für die Steuerung der spezifischen willkürmotorischen Handlung ist die sensorische Information wichtig. Der Integrationsleistung und ganz speziell der situationsabhängigen Gewichtung kommen damit nochmals eine besondere Bedeutung zu. Je geringer der gleichgewichtsrelevante Anteil und je geringer der visuelle Anteil, desto wichtiger ist die propriozeptive Information am motorischen Lernprozess.

Zwar sind die Informationen der verschiedenen Systeme unabhängig voneinander [12], werden aber zur Orientierung im Raum zentral integriert und beeinflussen sich gegenseitig [5, 15]. Auch das Bewusstsein über die Körperposition scheint die Repräsentation z. B. der Erdvertikalen zu modulieren [2].

Kinetosen Besonders deutlich wird die gemeinsame Verarbeitungsleistung dann, wenn es zu widersprüchlichen sensorischen Informationen kommt. Folgeerscheinungen werden z. B. unter dem Begriff der Kinetosen zusammengefasst, zu denen die Flug-, See- und Reisekrankheit zählen [20]. Eine besondere Bedeutung in der Verarbeitung sensorischer Information wird dabei dem Thalamus zugesprochen, der als Schaltstation zwischen afferenten Leitungsbahnen und der Großhirnrinde gilt [16].

\section{Propriozeption messen}

Zum somatosensorischen Sinnessystem zählen neben dem Berührungssinn, dem Schmerz, dem Juckreiz und der viszeralen Information auch die Propriozeption. Dieses Sinnessystem erfasst verschiedene Sinnesmodalitäten:
- Position der Körpersegmente und des Körpers

- Bewegung der Körpersegmente und des Körpers

- Grad der körperlichen Anstrengung

- Kraft

- Schweregefühl

Position und Bewegung Die beiden ersteren, Position und Bewegung, sind im Sport und auch in der klinischen Forschung die beiden meistuntersuchten Sinnesmodalitäten. Häufig werden diese allerdings nur sehr grob erfasst. Für den neurologischen Befund zum Beispiel erfolgt die Einteilung der Wahrnehmung häufig in nur drei Kategorien: normal, reduziert oder fehlend. In den letzten Jahren wurden daher verschiedene Geräte entwickelt, anhand derer die Propriozeption sogar gradgenau erfasst werden kann $[6,8,9]$. Bevor eine Testung durchgeführt wird, sollte aber nicht nur klar sein, welche der oben genannten Sinnesmodalitäten man adressiert, sondern auch, welcher Aspekt der jeweiligen Sinnesmodalität untersucht werden soll. Unterschieden werden hier zwei grundlegende Aspekte: Man sucht entweder nach der Schwelle, bei der ein Stimulus noch wahrnehmbar ist (Detektionsschwelle), oder nach der Schwelle, bei der zwei wahrnehmbare Stimuli noch unterschieden werden können (Diskriminationsschwelle) [13].

Robotergestützte Testoption Konczak und Mitarbeiter verwenden z. B. den sogenannten Wristbot, ein robotergestütztes Messsystem, mit dem die Propriozeption des Handgelenks erfasst werden kann [6]. Dieser Handgelenkroboter kann die drei Freiheitsgrade des Handgelenks ansprechen, das heißt, Bewegungen in jeder Gelenkachse zulassen, unterbinden oder durch entsprechende Aufschaltung von Kräften die Bewegungsausführung assistieren oder erschweren. Angetrieben über 4 Elektromotoren sind selektive Flexion/Extension, Ab-/Adduktion und Pro-/Supination oder aber Kombinationsbewegungen dieser drei Freiheitsgrade möglich. Zur Bestimmung der Diskriminationsschwelle der Handgelenkpositionen in Flexion/Extension wurden zwei passive Positionsstimuli präsentiert und mit der Forced-Choice-Methode überprüft. Das heißt, das Handgelenk des Probanden wurde durch den Wristbot in zwei unterschiedliche Handgelenkpositionen gebracht, von denen dann derjenige Stimulus zu benennen war, der am weitesten von der Neutral-Null-Stellung des Handgelenks entfernt war. Für eine einzige Testung wird so eine Reihe unterschiedlicher Stimuli-Paarungen präsentiert, bei denen die beiden Stimuli mal weiter und mal weniger weit voneinander abweichen. Man sucht in dieser Testung nach der kleinsten noch wahrnehmbaren Differenz, die richtig erkannt wird. Die computergestützte Testung ermöglicht es, über einen adaptiven Algorithmus die jeweils nächste Stimuli-Paarung und letztlich auch die sensorische Schwelle zu berechnen.

Gerätegestützte Testoption Eine andere Möglichkeit, Propriozeption zu messen, wird in der Studie von Holst- 


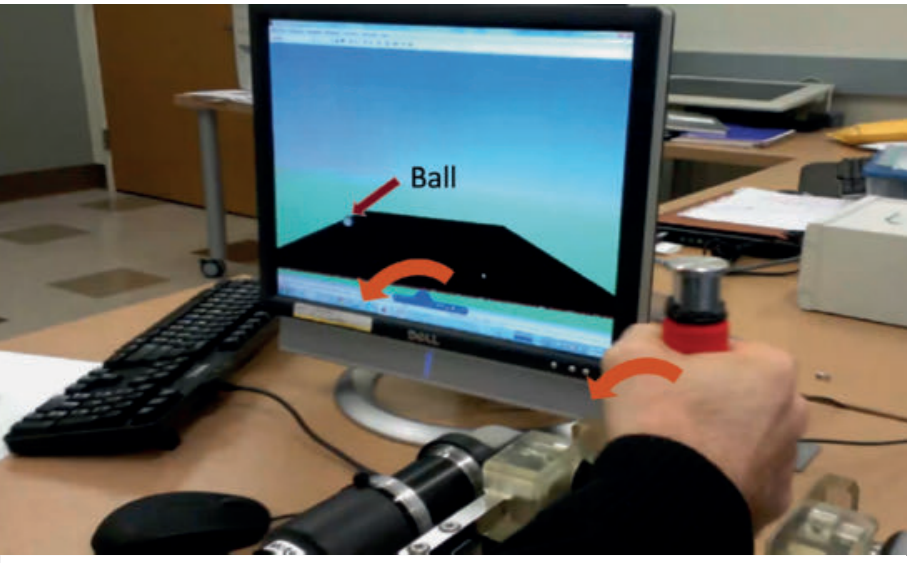

Abb. 2 Durch eine Flexionsbewegung des Handgelenks wird die virtuelle Tischplatte entgegen des Uhrzeigersinns gekippt, und der Ball bewegt sich nach links. (Quelle: C. Krewer)

Wolf et al. [10] beschrieben. In dieser Studie an 308 gesunden Kindern im Alter zwischen 5 und 17 Jahren wurde das bilaterale Messsystem Manipulandum verwendet. Es handelt sich hier um ein Testsetting, das verwendet wurde, um die Detektion der Ellbogenposition in Extension/Flexion zu untersuchen. Die Versuchsperson hat beide Unterarme auf beweglichen Schwenkelementen. Ein Schwenkarm wird durch den Untersucher in eine bestimmte Position gebracht, und die Versuchsperson soll den anderen Schwenkarm selbstständig durch Bewegung des Unterarms, der sich auf diesem Schwenkarm befindet, matchen (also in die gleiche, spiegelverkehrte Position bringen). Je fünf Durchgänge wurden für drei unterschiedliche Positionen durchgeführt, und so wurde der Positionsfehler, also die Abweichung zur vorgegebenen Ellbogenposition, bestimmt.

Vergleich In beiden Beispielen wird die Modalität „Position" gemessen. Beide Beispiele unterscheiden sich jedoch in drei Aspekten: Im Vergleich zum ersten Beispiel wird im zweiten Testsetting nur ein Stimulus präsentiert (Diskrimination versus Detektion), dessen richtiges Erkennen durch eine aktive Eigenbewegung (passive versus aktive Testung) im gleichen Gelenk der anderen Körperseite angezeigt wird (unilateral versus bilateral). Diese drei Aspekte können je Testung der Propriozeption variiert werden.

\section{WICHTIG}

Egal welche dieser beiden Methoden für die Testung verwendet wird - wichtig ist, dass die Testung der Propriozeption unter Ausschluss visueller Information stattfindet, um so sicherzustellen, dass die relevante Information über die propriozeptiven Rezeptoren aufgenommen wird.

\section{Neurologische Rehabilitation}

Neurologische Erkrankungen können Störungen oder sogar komplette Ausfälle sensorischer Informationen verursachen. Diese Leistungseinschränkung kann durch eine Schädigung der peripheren und oder der zentralen Komponenten des jeweiligen Sinnessystems bedingt sein. Zum einen können also Aufnahme (Rezeptoren) und Weiterleitung (Neurone) der Informationsverarbeitung beeinträchtigt sein und zum anderen auch die nichtattentionalen und attentionalen zentralen Verarbeitungsanteile. Nichtattentionale Anteile betreffen die impliziten Aspekte des sensomotorischen Lernens [1], die attentionalen Anteile kommen bei der Wahrnehmung, also der Perzeption der Sinnesinformation, zum Tragen.

Umgewichtung der sensorischen Information Für die neurologische Rehabilitation sind folgende Phänomene interessant: Führt z. B. eine bilaterale Vestibulopathie zu einem peripheren Ausfall der vestibulären Information, kommt es zu einer sensorischen Umgewichtung (engl.: sensory reweighting). Medendorp et al. [17] führten bei Patienten mit und ohne bilaterale Vestibulopathie den Stab-Rahmen-Test (engl.: Rod and Frame Test) durch. Der Stab-Rahmen-Test ist eine Raumorientierungsaufgabe, bei der ein leuchtender Stab und ein leuchtender rechteckiger Rahmen in einem abgedunkelten Raum präsentiert werden. Sowohl Stab als auch Rahmen sind beide verkippt. Auch die Kopfposition wird für diese Aufgabe variiert. Aufgabe ist es nun, den Stab zu repositionieren und senkrecht zur Erdvertikalen zu orientieren. Die Autoren konnten zeigen, dass Patienten mit bilateraler Vestibulopathie im Vergleich zu gesunden Kontrollpersonen einen größeren Bias und eine größere Variabilität in der Vertikalenwahrnehmung haben. Unter Berücksichtigung des variierenden Testsettings kommen die Autoren zu dem Schluss, dass die Patienten zur Bearbeitung der Aufgabe stärker die visuellen Informationen nutzen als die gesunden Kontrollpersonen, die sich stärker auf die vestibuläre Information stützen, und interpretieren dies als Umgewichtung der sensorischen Information.

Willkürmotorisches Bewegungstraining Ein weiteres Phänomen, das bereits in mehreren Studien beschrieben ist, ist bei zentralen Erkrankungen zu beobachten. Durch ein willkürmotorisches Bewegungstraining lässt sich neben der motorischen Funktion auch die propriozeptive Funktion verbessern. Bei Patienten mit idiopathischem Parkinson-Syndrom konnte dies für die Funktionen des Handgelenks nachgewiesen werden. Elangovan et al. [6] trainierten 13 an Parkinson erkrankte Erwachsene mit dem Handgelenkroboter Wristbot. Neben der Anwendung als propriozeptives Messinstrument kam in dieser Studie der Roboter auch für die motorische Intervention zur Anwendung ( $\triangleright$ Abb. 2). Durch kleinamplitudige Flexion-Extensions-Bewegungen wird ein virtueller Ball auf einer virtuellen Tischplatte ins Rollen gebracht, um den Ball kontrolliert über ein fixes Zielareal zu bringen. Schafft eine Testperson 
es, das Zielareal für 5 Sekunden zu halten, wird ein neues Zielareal angezeigt. Je nach Funktionslevel des Patienten können verschiedene Schwierigkeitslevel und verschiedene Arbeitsbereiche für Flexion/Extension definiert werden. Für die Studie wurden insgesamt 60 Zielareale festgelegt.

Vor und nach dieser Therapieeinheit mit 60 Zielarealen wurden jeweils verschiedene Tests durchgeführt, u. a. die motorische Lernleistung in ebendieser Aufgabe und die Propriozeption (s. o.). Die Patienten verbesserten sich sowohl in der motorischen als auch in der Handgelenk-Positions-Diskriminationsaufgabe. Ein Transfer dieser Verbesserungen auf einen Handschreibtest konnte in dieser Studie allerdings nicht nachgewiesen werden.

Stimulation der Fingerkuppen Andere Verfahren fokussieren speziell auf die Erhöhung der sensorischen Information mit dem Ziel, den motorischen Outcome zu verbessern. Ein Beispiel für diese therapeutische Herangehensweise ist die elektrische Stimulation der Fingerkuppen mit dem Therapiehandschuh tipstim ( $\triangleright$ Abb. 3). Während dieser Stimulation ruht die Hand auf einer stabilen Unterlage. Es findet zeitgleich keine Bewegung statt.

Kattenstroth et al. [11] behandelten Patienten mit sensomotorischen Defiziten der Hand drei bis vier Wochen nach Schlaganfall mit diesem therapeutischen Ansatz im Rahmen einer randomisierten kontrollierten Studie. Die elektrische $20-\mathrm{Hz}$-Stimulation wurde dabei an der paretischen Hand für 45 Minuten täglich, fünf Tage die Woche über einen Zeitraum von zwei Wochen appliziert. Vor und nach der Interventionsphase wurden verschiedene Tests zur Erfassung der Veränderung durch die Therapie vorgenommen. Die Testverfahren und Assessments wurden von den Autoren in jeweils vier funktionellen Domänen eingeteilt: Sensorik, Motorik, Propriozeption und Alltagsaktivität. Der sensorischen Domäne wurden zwei Testungen zur Erfassung der taktilen Funktion der Zeigefingerkuppe zugeordnet (Frey-Filamente und Grating Orientation Task), der motorischen Domäne wurden zwei Testungen zur Erfassung der Handgriffkraft und der Feinmotorik (Dynamometer und Nine Hole Peg Task) zugeordnet, der Domäne Propriozeption die Erfassung der Wahrnehmung der Gelenkposition (Joint Position Sense) und die Alltagsaktivitäten wurden mit dem Jebsen-Taylor-Handtest erfasst. Die Propriozeption wurde in dieser Studie mit dem Bochum Joint Position Sense Assessment erfasst. Für diese Testung werden von der Testperson Styroporkugeln in sieben verschiedenen Größen (3, 5, 6, 7, 8, 10 und $12 \mathrm{~cm})$ in der paretischen Hand gehalten. Eine Referenzkugel hält die Testperson in der nichtparetischen Hand. Für jede Paarung erfolgt dann eine Einschätzung der Kugelgröße für die in der paretischen Hand gehaltene Kugel. Eingeschätzt werden soll, ob die Kugel im Vergleich zur Referenzkugel kleiner, größer oder gleich groß ist.

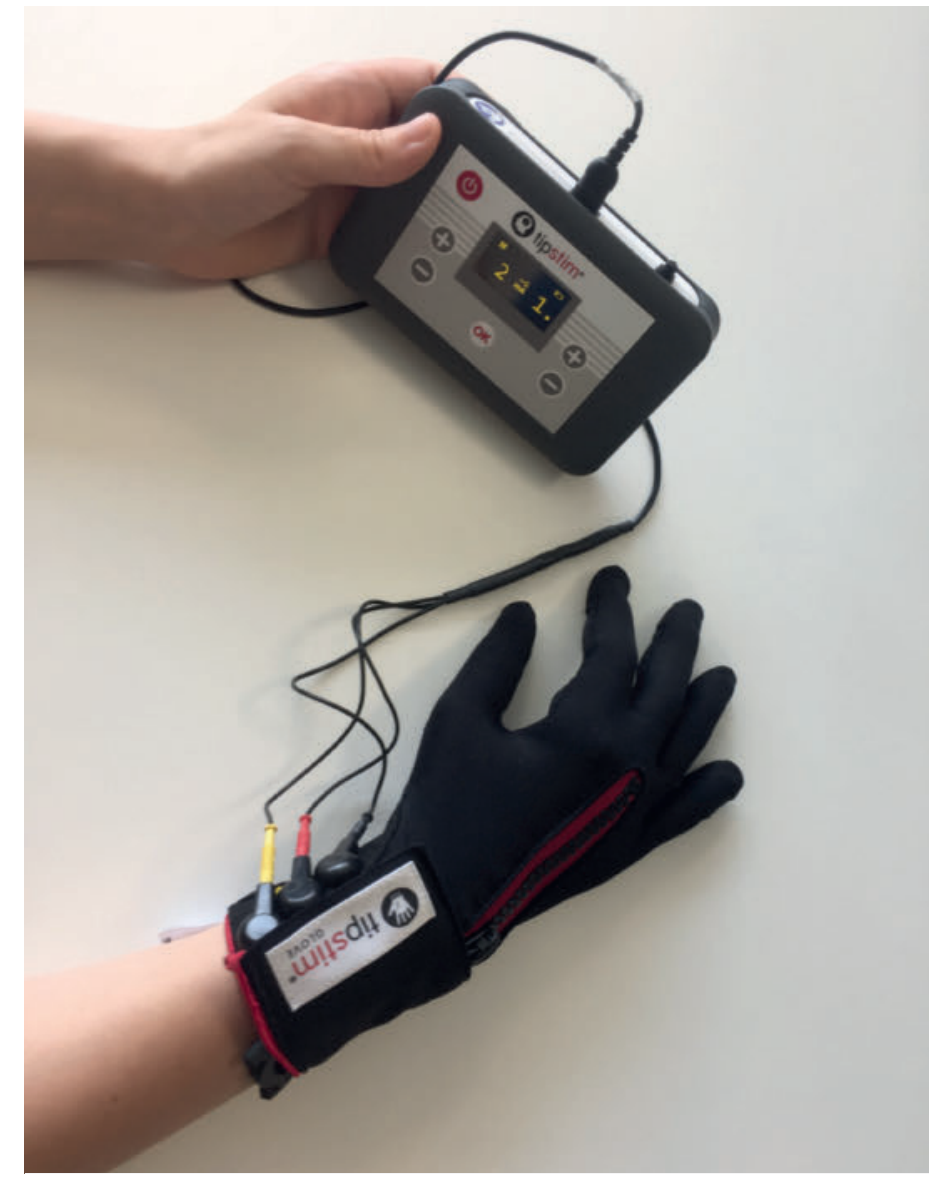

Abb. 3 Sensible Stimulation der Fingerspitzen mittels Therapiehandschuh tipstim ${ }^{\circledR}$ (Bosana Medizintechnik GmbH, Dorsten, Deutschland; Quelle: C. Krewer)

Die Autoren konnten zeigen, dass die Interventionsgruppe, die zusätzlich die sensorische Stimulation erhalten hatte, in allen vier Domänen einen besseren Outcome hatte als die Kontrollgruppe, die keine sensorische Stimulation erhalten hatte. Am deutlichsten waren die Verbesserungen in den beiden Domänen Sensorik (also in dieser Forschungsarbeit die taktile Wahrnehmung) und Motorik.

\section{Schlussbetrachtung}

Diese Studien zeigen, dass die Intensivierung einer spezifischen sensorischen Information im Rahmen eines neurorehabilitativen Konzepts das motorische Lernen unterstützt, es also zu einem verbesserten motorischen Outcome kommen kann. Es zeigt sich aber auch, dass primär motorische Interventionen einen positiven Effekt auf die sensorische Wahrnehmungsleistung haben. Je besser die Verfahren zur Erfassung der somatosensorischen Information werden und je gezielter sie zum Einsatz kommen, desto detaillierter kann dieser Zusammenhang von motorischer und somatosensorischer Funktion zukünftig untersucht werden. Für den interessierten Leser sei an dieser Stelle auf das Review von Aman et al. [1] verwiesen, das einen guten Über- 
blick über verschiedene Verfahren bietet, die speziell die Verbesserung der Propriozeption avisieren.

\section{Autorinnen/Autoren}

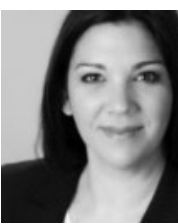

\section{Dr. Carmen Krewer}

Bewegungswissenschaftlerin; Forschungsabteilung der Schön Klinik Bad Aibling: Schwerpunkt motorische Kontrolle/ motorisches Lernen in der Neurorehabilitation. Assoziierte wissenschaftliche Mitarbeiterin am Lehrstuhl für Bewegungswissenschaft der Fakultät für Sport- und Gesundheitswissenschaften der TU München.

\section{Korrespondenzadresse}

\section{Dr. Carmen Krewer}

Schön Klinik Bad Aibling SE \& Co. KG

Kolbermoorer Straße 72

83043 Bad Aibling

E-Mail: ckrewer@schoen-klinik.de

www.schoen-klinik.de

\section{Literatur}

[1] Aman JE, Elangovan N, Yeh I-L et al. The effectiveness of proprioceptive training for improving motor function: $\mathrm{A}$ systematic review. Frontiers in Human Neuroscience 2015; 8: 1075

[2] Barra J, Pérennou D, Thilo KV et al. The awareness of body orientation modulates the perception of visual vertical. Neuropsychologia 2012. Im Internet: http://dx.doi. org/10.1016/j.neuropsychologia. 2012.06.021; Stand: 04.07.2019

[3] Blischke K. Entwicklung der Haltungskontrolle. In: Munzert J, Schott N, Hrsg. Motorische Entwicklung. Göttingen: Hogrefe; 2010: 30-48

[4] Blischke K, Schott N. Haltungskontrolle im höheren Lebensalter. Munzert J, Schott N, Hrsg. Motorische Entwicklung. Göttingen: Hogrefe; 2010: 49-68

[5] Dieterich M. Vestibuläres System und Störungen der vestibulären Raumorientierung. In: Karnath HO, Thier P, Hrsg. Neuropsychologie. 2. Aufl. Berlin: Springer; 2006: 197-205

[6] Elangovan N, Tuite PJ, Konczak J. Somatosensory training improves proprioception and untrained motor function in Parkinson's disease. Frontiers in Neurology 2018; 9: 1053. doi:10.3389/fneur.2018.01053

[7] Gardner EP, Johnson KO. Sensory coding. In: Kandel ER, Jessel TM, Schwartz JH, Siegelbaum SA, Hudspeth A], eds.
Principles of Neural Science. 5th ed. New York: McGraw-Hill; 2013: 449-474

[8] Gorst T, Freeman J, Yarrow K et al. Assessing lower limb position sense in stroke using gradient discrimination test (GrasDTTM) and step-height discrimination test (StepDTTM): A reliability and validity study. Disability and Rehabilitation 2019; 14: 1-9

[9] Han J, Waddington G, Adams R et al. Assessing proprioception: A critical review of methods. Journal of Sport and Health Science 2016; 5 (1): 80-90

[10] Holst-Wolf JM, Yeh IL, Konczak J. Development of proprioceptive acuity in typically developing children: Normative data on forearm position sense. Front Hum Neurosci 2016; 10: 436. doi:10.3389/fnhum.2016.00436

[11] Kattenstroth JCh, Kalisch T, Sczesny-Kaiser M et al. Daily repetitive sensory stimulation of the paretic hand for the treatment of sensorimotor deficits in patients with subacute stroke: RESET, a randomized, sham-controlled trial. BMC Neurology 2018; 18: 2. doi:10.1186/s12883-017-1006-z.

[12] Kelly JP. The sense of balance. In: Kandel ER, Schwartz JH, Jessell TM, eds. Principles of neural science. 3rd ed. Connecticut: Appelton \& Lange; 1991: 500-511

[13] Krewer C, Van de Winckel A, Elangovan $\mathrm{N}$ et al. Commentary on: „Assessing proprioception: A critical review of methods” by Han J, Waddington G, Adams R et al. Journal of Sport and Health Science 2016; 5: 91-92

[14] Lee DN, Aronson E. Visual proprioceptive control of standing in human infants. Perception and Psychophysics 1974; 15: $529-532$

[15] Lewald J. Auditives Orientieren im Raum und seine Störungen. In: Karnath HO, Thier P, Hrsg. Neuropsychologie. 2. Aufl. Berlin: Springer; 2006: 185-196

[16] Martin JH. Coding and processing of sensory information. In: Kandel ER, Schwartz JH, Jessell TM, eds. Principles of neural science. 3rd ed. Connecticut: Appelton \& Lange; 1991: 329-340

[17] Medendorp WP, Alberts BBGT, Verhagen WIM et al. Psychophysical evaluation of sensory reweighting in bilateral vestibulopathy. Front Neurol 2018; 9: 377

[18] Mittelstaedt H. Origin and processing of postural information. Neuroscience and Biobehavioral Review 1998; 22 (4): 473-478

[19] Ostry D], Gribble PL. Sensory plasticity in human motor learning. Trends in Neurosciences 2016; 39(2): 114-123. doi:10.1016/j.tins.2015.12.006

[20] Stoll W, Tegenthoff M. Schwindel und Gleichgewichtsstörungen. Stuttgart: Thieme; 2004

Bibliografie

DOI https://doi.org/10.1055/a-0980-2191

neuroreha 2019; 11: 112-116

(c) Georg Thieme Verlag KG Stuttgart · New York ISSN 1611-6496 